\title{
ANALYSIS OF HEAT TRANSFER BETWEEN AN INERT GAS AND A POROUS STRUCTURE WITH ULTRALOW THERMAL CONDUCTIVITY USING THE SUMUDU TRANSFORM METHOD
}

\author{
Gbeminiyi Sobamowo \\ Department of Mechanical Engineering, University of Lagos, Akoka, Lagos, Nigeria \\ mikegbeminiyiprof@yahoo.com
}

Received: 20 March 2018; Accepted: 5 August 2019

\begin{abstract}
The relatively new integral transform called the Sumudu transform method can be used to solve partial differential equations with variable coefficients and as well as intricate problems in engineering and applied mathematics without resorting to a new frequency domain. Unlike the other integral transforms, the Sumudu transform has scale and unit-preserving properties. However, the method is still not widely known or used for solving differential equations especially in the area of applied mathematics and engineering. As a means of demonstrating the potency of the method, the paper applied the Sumudu transform to present analytical solutions of a one-dimensional problem of heat transfer between an inert gas and an ultralow thermal conductivity porous medium. The developed analytical solutions are used to investigate the heat propagation in the porous medium. Depending on the initial temperature, it is established from the study that there are snapshots of the heat wave propagating and a sharp heat front propagation through the medium during its heating or cooling. This sharp front is difficult to detect and quantify by numerical methods. Hence, exact analytical solutions are presented in this study. As it is demonstrated in this study, it is hoped that the Sumudu transform method will be applied to other various complex engineering problems.
\end{abstract}

MSC 2010: $35 A 15$

Keywords: Sumudu transform method, heat transfer, inert gas, porous structure, ultralow thermal conductivity

\section{Introduction}

The recent advancements in materials studies show the importance and various applications of nanoporous materials with ultralow thermal conductivity. Indisputably, the nanoporous materials have excellent thermal insulation due to their ultralow thermal conductivities. Consequently, they have been applied in various industrial and engineering processes such as high temperature energy storage packed beds [1], capillary electrochromatography [2], or food engineering [3] 
where the heat transfer between an inert gas and a low thermal conductivity porous material are demonstrated. Also, the study of mechanical and thermal behaviours of ultralow thermal conductivity nanostructured materials, such as thin films, superlattices, nanowires and nanocomposites have been the research focus of a large number of recent studies $[4,5]$. Such ultralow thermal conductivity nanostructured materials present a thermal conductivity only a few percent larger than that of air, being smaller than the corresponding theoretical lower bound for a solid, referred to as Einstein limit [6]. In order to provide good physical insights into thermomechanical behaviours of the material and also to study the significance of various system parameters affecting the mechanical and thermal behaviours of the material, various theoretical studies have been carried out [7-11]. The solutions of the full set of nonlinear partial differential equations for the present problem under both constant [7] and variable [8] porosity have been obtained by numerical methods [9]. However, the numerical methods are inherent with high computational cost and time. Moreover, the validation of the numerical methods is simplified when a set of simple analytical solutions is known.

Therefore, the classical way of finding an exact analytical solution is obviously still very important since it serves as an accurate benchmark for the numerical solutions. Also, the experimental data are useful to access the mathematical models, but are never sufficient to verify the numerical solutions of the established mathematical models. When exact analytical solutions are available, they provide good insights into the significance of various system parameters affecting the phenomena as it gives continuous physical insights rather than pure numerical or computation methods. An exact analytical expression is more convenient for engineering calculations compare with experimental or numerical studies and it is an obvious starting point for a better understanding of the relationship between physical quantities/ properties. It is convenient for parametric studies, accounting for the physics of the problem and appears more appealing than the numerical solutions. Development of exact analytical solutions for differential equations involves the applications of integral transforms (Laplace, Fourier, Mellin, Hankel, etc.), the method of separation of variables, the method of parameters variation, Green functions, etc. However, in recent times, a new integral transform method called Sumudu transform was originally proposed by Watugala $[12,13]$, its properties were established by Asiru [14-16]. Subsequently, its principle, procedure and applications were exploited by Weerakoon $[17,18]$. As a means of demonstrating its potency along side its appealing properties, Eltayeb and Kilicman [19-21] and others researchers [22-24] have applied the method to solve various ordinary and partial differential equations. It was shown that the method has scale and unit-preserving properties and can be used to solve partial differential equations with variable coefficients and also, intricate problems in engineering mathematics and applied sciences without resorting to a new frequency domain. Nevertheless, the new integral transform is still not widely known or used in engineering and applied mathematics.

The Sumudu transform method is applied to the present problem under investigation. In this paper, the method is used to develop analytical solutions of the heat 
transfer between an inert gas and a porous matrix. The analytical solutions are used to investigate the heat propagation in the porous medium. The developed analytical solutions of the heat transfer between a non-reactive gas flow through a porous medium gives great physical insight into the dynamics of the system.

\section{Mathematical model and the assumptions}

Let us consider the heat transfer between a gas and a porous solid, long enough to be considered as semi-infinite in extent, under the following assumptions: the gas is an incompressible Newtonian fluid, with negligible viscous dissipation, negligible heat conduction among the fluid particles, and the fluid motion is only in the axial direction of the solid, from the inlet to the outlet, in quasi-steady conditions; the solid has a constant porosity and negligible radial temperature gradient, with only an axial temperature gradient. This two-phase problem is modelled through the system of coupled partial differential equations [11]:

$$
\begin{gathered}
\varepsilon_{f} \rho_{f} c_{f}\left(\frac{\partial T_{f}}{\partial t}+v_{f} \frac{\partial T_{f}}{\partial x}\right)=h\left(T_{s}-T_{f}\right) \\
\left(1-\varepsilon_{f}\right) \rho_{s} c_{s} \frac{\partial T_{s}}{\partial t}=\left(1-\varepsilon_{f}\right) k_{s} \frac{\partial^{2} T_{f}}{\partial x^{2}}+h\left(T_{f}-T_{s}\right)
\end{gathered}
$$

Initial condition (both phases start in thermal equilibrium at $T_{o}$ )

$$
t=0, \quad \mathrm{~T}_{f}=T_{s}=T_{o}, x>0
$$

Boundary conditions:

$$
\begin{gathered}
t>0, x=0, \quad \mathrm{~T}_{f}=T_{f o} \\
t>0, \lim _{x \rightarrow \infty} T_{f}(x, t)=\lim _{x \rightarrow \infty} T_{s}(x, t)=T_{o}
\end{gathered}
$$

where $x$ is the axial position along the medium, $t$ is time, the subscripts $f$ and $s$ stand for the fluid and the solid matrix, respectively, $T$ is the temperature, $q$ is the density, $c_{s}$ is the specific heat of the solid, $c_{f}$ is the specific heat at constant pressure of the fluid, $v_{f}$ is the fluid flow velocity, $h$ is the heat transfer coefficient between the fluid and the solid, $k_{s}$ is the thermal conductivity, and $\varepsilon$ is the effective porosity.

It should be pointed out that for an ultra low thermal conductivity solid, $k_{s}$ has a very small value that approaches zero. Also, the convective heat transfer domnates the heat transfer in the fluid/gas to the point that the conductive heat transfer is negligible. Therefore, Eqs. (1) and (2) become: 


$$
\begin{gathered}
\varepsilon_{f} \rho_{f} c_{f}\left(\frac{\partial T_{f}}{\partial t}+v_{f} \frac{\partial T_{f}}{\partial x}\right)=h\left(T_{s}-T_{f}\right) \\
\varepsilon_{s} \rho_{s} c_{s} \frac{\partial T_{s}}{\partial t}=h\left(T_{f}-T_{s}\right)
\end{gathered}
$$

and the initial and the boundary conditions become

$$
\begin{gathered}
t=0, \mathrm{~T}_{f}=T_{s}=T_{o}, \quad x>0 \\
t>0, \quad x=0, \quad \mathrm{~T}_{f}=T_{f o}
\end{gathered}
$$

Equations (5)-(8) can be nondimensionalized by using the following dimensionless variables

$$
\theta_{f}=\frac{T_{f}-T_{o}}{T_{f_{o}}-T_{o}}, \theta_{s}=\frac{T_{s}-T_{o}}{T_{f_{o}}-T_{o}}, \tau=\frac{h t}{\varepsilon_{s} \rho_{s} c_{s}}, X=\frac{\beta h x}{\varepsilon_{f} \rho_{f} c_{f} v_{f}}, \beta=\frac{\varepsilon_{f} \rho_{f} c_{f}}{\varepsilon_{s} \rho_{s} c_{s}}
$$

The dimensionless forms of Eqs. (5) and (6) are

$$
\begin{gathered}
\beta\left(\frac{\partial \theta_{f}}{\partial \tau}+\frac{\partial \theta_{f}}{\partial X}\right)=\theta_{s}-\theta_{f} \\
\frac{\partial \theta_{s}}{\partial \tau}=\theta_{f}-\theta_{s}
\end{gathered}
$$

the dimensionless initial condition is given as

$$
\tau=0, \quad \theta_{f}=0, \quad \theta_{s}=0, X>0
$$

and the boundary condition in nondimensional form is

$$
\tau>0, \quad X=0, \quad \theta_{f}=1
$$

Applying the Sumudu transform to Eqs. (10)-(12), one arrives at

$$
\begin{gathered}
\beta\left(\frac{1}{u} \bar{\theta}_{f}\left(\frac{1}{u}, X\right)+\frac{d \bar{\theta}_{f}(u, x)}{d X}\right)=\bar{\theta}_{s}\left(\frac{1}{u}, X\right)-\bar{\theta}_{s}\left(\frac{1}{u}, X\right) \\
\frac{1}{u} \theta_{s}\left(\frac{1}{u}, X\right)=\bar{\theta}_{f}\left(\frac{1}{u}, X\right)-\bar{\theta}_{s}\left(\frac{1}{u}, X\right)
\end{gathered}
$$


The boundary condition in the Sumudu domain

$$
\theta_{f}=1, \quad X=0, u>0
$$

From Eq. (15)

$$
\bar{\theta}_{s}\left(\frac{1}{u}, X\right)=\frac{\theta_{f}\left(\frac{1}{u}, X\right)}{\left(\frac{1}{u}+1\right)}
$$

On substituting Eq. (17) into Eq. (14), we have

$$
\frac{\beta}{u} \bar{\theta}_{f}\left(\frac{1}{u}, X\right)+\beta \frac{d \bar{\theta}_{f}\left(\frac{1}{u}, X\right)}{d X}=\left[\left(\frac{1}{\frac{1}{u}+1}\right)-1\right] \bar{\theta}_{f}\left(\frac{1}{u}, X\right)
$$

A further simiplification of Eq. (18) results in

$$
\frac{\beta}{u} \bar{\theta}_{f}\left(\frac{1}{u}, X\right)+\beta \frac{d \bar{\theta}_{f}\left(\frac{1}{u}, X\right)}{d X}=-\frac{1}{u}\left(\frac{1}{\frac{1}{u}+1}\right) \bar{\theta}_{f}\left(\frac{1}{u}, X\right)
$$

Equation (19) can also be written as

$$
\beta \frac{d \bar{\theta}_{f}\left(\frac{1}{u}, X\right)}{d X}+\frac{1}{u}\left(\beta+\frac{1}{\frac{1}{u}+1}\right) \bar{\theta}_{f}\left(\frac{1}{u}, X\right)=0
$$

or

$$
\frac{d \bar{\theta}_{f}\left(\frac{1}{u}, X\right)}{d X}+\frac{1}{u}\left(1+\frac{1}{\beta\left(\frac{1}{u}+1\right)}\right) \bar{\theta}_{f}\left(\frac{1}{u}, X\right)=0
$$

After integration of Eq. (21), the equation becomes

$$
\bar{\theta}_{f}\left(\frac{1}{u}, X\right)=e^{-\frac{X}{u}\left(1+\frac{1}{\beta\left(\frac{1}{u}+1\right)}\right)}
$$


On substituting Eq. (22) into Eq. (17), we have

$$
\bar{\theta}_{s}\left(\frac{1}{u}, X\right)=\frac{e^{-\frac{X}{u}\left(1+\frac{1}{\beta\left(\frac{1}{u}+1\right)}\right)}}{\frac{1}{u}+1}
$$

Applying the inverse Sumudu transform to Eq. (22) and (23) with the application of convolution theorem, using the Bromwich integral inversion Theorem, one arrives at:

$$
\begin{gathered}
\bar{\theta}_{f}(\tau, X)=\mathrm{H}(\tau-X) e^{-\frac{X}{\beta}}\left[1+\int_{0}^{\tau-X} e^{-p} I_{1}\left(2 \sqrt{\frac{X p}{\beta}}\right) \sqrt{\frac{X}{\beta p}} d p\right] \\
\bar{\theta}_{f}(\tau, X)=\mathrm{H}(\tau-X) e^{-\frac{X}{\beta}} \int_{0}^{\tau-X} e^{-p} I_{0}\left(2 \sqrt{\frac{X p}{\beta}}\right) d p
\end{gathered}
$$

where $\mathrm{H}(u)$ is the Heavisides unit step function. Eqs. (24) and (25) can be numerically evaluated using Simpson's, trapezoidals, Weddle's rule or by means of numerical quadrature rule such as the adaptive Gauss-Kronrod quadrature.

Preferably, one can transform Eqs. (24) and (25) numerically by adopting Simon's approach as used in the inverse Laplace transform to inverse the Sumudu transform:

$$
\begin{aligned}
& \theta_{f}(\tau, X)=\frac{e^{a_{p} \tau}}{\tau}\left[\frac{1}{2} \bar{\theta}_{f}\left(a_{p}, X\right)+\operatorname{Re} \sum_{n=1}^{N} \bar{\theta}_{f}\left(a_{p}+i \frac{n \pi}{t}, X\right)(-1)^{n}\right] \\
& \theta_{s}(\tau, X)=\frac{e^{a_{p} \tau}}{\tau}\left[\frac{1}{2} \bar{\theta}_{s}\left(a_{p}, X\right)+\operatorname{Re} \sum_{n=1}^{N} \bar{\theta}_{s}\left(a_{p}+i \frac{n \pi}{t}, X\right)(-1)^{n}\right]
\end{aligned}
$$

where:

$$
\begin{aligned}
& \bar{\theta}_{s}\left(a_{p}+i \frac{n \pi}{t}, X\right)=\frac{e^{-X\left(a_{p}+i \frac{n \pi}{t}\right)\left\{1+\frac{1}{\beta\left[\left(a_{p}+i \frac{n \pi}{t}\right)+1\right]}\right\}}}{\left[\left(a_{p}+i \frac{n \pi}{t}\right)+1\right]}, \\
& \bar{\theta}_{f}\left(a_{p}+i \frac{n \pi}{t}, X\right)=e^{-\left[X\left(a_{p}+i \frac{n \pi}{t}\right)\left\{1+\frac{1}{\beta\left[\left(a_{p}+i \frac{n \pi}{t}\right)+1\right]}\right\}\right]},
\end{aligned}
$$


The above Eqs. (26) and (27) are the models of the heat convection through the porous medium, the boundary condition at $x=0$ generates a temperature wavefront propagating at constant velocity, equal to unity in nondimensional units.

\section{Results and discussion}

The analysis in the previous section shows the use of the Sumudu transform in the development of exact analytical solutions to the model of a one-dimensional problem of heat transfer between an inert gas and an ultralow thermal conductivity porous medium. The results of the analysis of temperature profiles for the gas and solid are show in Figures 1 and 2.

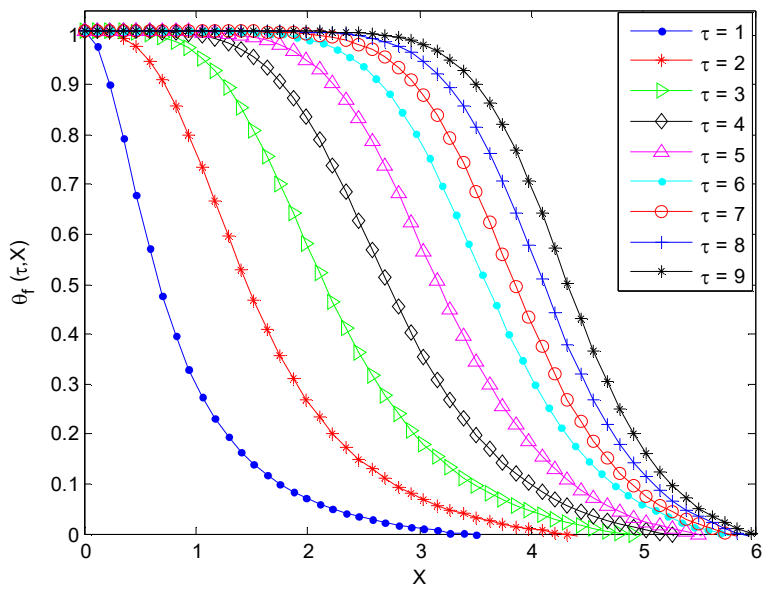

Fig. 1. Evolution of the profiles of the temperatures of the gas for $\beta=0.5$ at different times

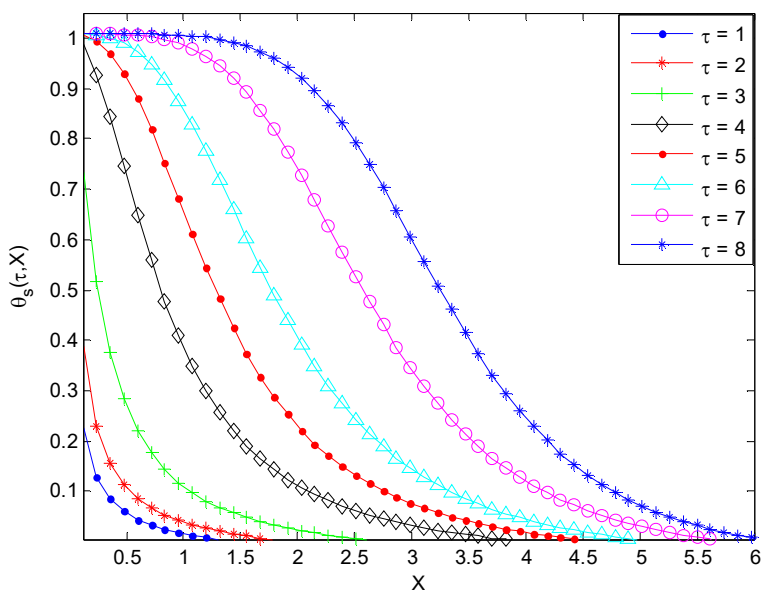

Fig. 2. Evolution of the profiles of the temperatures of the solid matrix for $\beta=0.5$ at different times 
Figures 1 and 2 show the temporal evolution of the dimensionless temperatures $\theta_{f}(\tau, X)$ and $\theta_{s}(\tau, X)$, respectively. The figure shows snapshots of the heat wave propagating from the left to the right corresponding to the heating of the solid by the gas. Figures 1 and 2 show the temperature profiles for the gas and solid, respectively, for $\beta=0.5$, corresponding to a situation in which the gas (solid) decreases (increases) its temperature faster (slower) than as $\beta$ increases. Figure 1 shows that the height of the gas front decreases quickly, resulting in a temperature profile smoother and more similar to that of a diffusive equation. Similarly, Figure 2 shows that the profile of the solid temperature also resembles that of a diffusive front, showing a spatial derivative rapidly approaching to zero. The temperature of the porous medium at the inlet increases until reaching that of the incoming gas flow, equal to unity in non-dimensional variables. As $n$ increases (not shown), a sharp discontinuous front propagating at unit speed is noticed. This sharp front presented by the analytical method is difficult to detect and quantify by numerical methods. Therefore, the present solution can be used to validate numerical schemes for heat transfer between fluids and porous media with negligible or non-negligible thermal conductivity. Also, in the present work, Sumudu transform method is used and the same results were obtained as in the previous work [11]. Although the data of the past work were not available for comparison, a close look at the results in the present work and the work of Pérez et al. [11] shows very good agreements. The results in the present work are developed as benchmarks for the future results on the same problem.

\section{Conclusions}

The analytical solutions of the problem of heat transfer between an inert gas and an ultralow thermal conductivity porous solid have been obtained using Sumudu transform method. The results of the temporal evolution of the dimensionless temperatures of the gas and solid matrix have been provided graphically. Also, the inherent snapshots of the heat wave propagating and a sharp heat front propagation through the medium during its heating or cooling which are very difficult to be detected and quantified by numerical methods are detected and quantified by exact analytical solutions using Sumudu transforms. The present solutions can be used to validate numerical schemes for heat transfer between fluids and porous media with negligible or non-negligible thermal conductivity.

\section{References}

[1] Andrepont, J.S. (2003). Thermal energy storage: solutions for demand management. Energy Eng., $100,66-80$.

[2] Nischang, I., \& Tallarek, U. (2007). Fluid dynamics in capillary and chip electrochromatography. Electrophoresis, 28, 611-626.

[3] Kostaropoulos, A.E., \& Saravacos, G.D. (1997). Thermal diffusivity of granular and porous foods at low moisture content. J. Food Eng., 33, 101-109. 
Analysis of heat transfer between an inert gas and a porous structure with ultralow thermal conductivity ...87

[4] Prasher, R. (2006). Ultralow thermal conductivity of a packed bed of crystalline nanoparticles: a theoretical study. Phys. Rev. B, 74, 165413.

[5] Chiritescu, C., Cahill, D.G., Nguyen, N., Johnson, D., Bodapati, A., Keblinski, P., \& Zschack, P. (2007). Ultralow thermal conductivity in disordered, layered WSe2 crystals. Science, 315, 351-353.

[6] Cahill, D.G., Watson, S.K., \& Pohl, R.O. (1992). Lower limit to the thermal conductivity of disordered crystals. Phys. Rev. B, 46, 6131-6140.

[7] Awartani, M., \& Hamdan, M.H. (1999). Non-reactive gas-particulate models of flow through porous media. Appl. Math. Comput., 100, 93-102.

[8] Allan, F.M., Qatanani, N., Barghouthi, I., \& Takatka, K.M. (2004). Dusty gas model of flow through naturally occurring porous media. Appl. Math. Comput., 148, 809-821.

[9] Siyyam, H., Merabet, N., \& Hamdan, M.H. (2007). Standard numerical schemes for coupled parallel flow over porous layers. Appl. Math. Comput., 194, 38-45.

[10] Awartani, M.M., \& Hamdan, M.H. (2005). Fully developed flow through a porous channel bounded by flat plates. Appl. Math. Comput., 169, 749-757.

[11] Pérez, J., Villatoro, F.R., Santander, J.L.G., Borovsky, M.A., Ratis, Yu.L., Izzheurov, E.A., \& Fernández de Córdoba, P. (2008). Heat transfer between a gas and an ultralow thermal conductivity porous structure. Applied Mathematics and Computation, 204, 687-693.

[12] Watugala, G.K. (1993). Sumudu transform: a new integral transform to solve differential equations and control engineering problems. International Journal of Mathematical Education in Science and Technology, 24(1), 35-43.

[13] Watugala, G.K. (1998). Sumudu transform new integral transform to solve differential equations and control engineering problems. Mathematical Engineering in Industry, 6(4), 319-329.

[14] Asiru, M.A. (2002). Further properties of the Sumudu transform and its applications. International Journal of Mathematical Education in Science and Technology, 33(3), 441-449.

[15] Asiru, M.A. (2002). Sumudu transform and the solution of integral equations of convolution type. International Journal of Mathematical Education in Science and Technology, 32(6), 906-910.

[16] Asiru, M.A. (2003). Classroom note: application of the Sumudu transform to discrete dynamic systems. International Journal of Mathematical Education in Science and Technology, 34(6), 944-949.

[17] Weerakoon, S. (1994). Applications of Sumudu transform to partial differential equations. International Journal of Mathematical Education in Science and Technology, 25(2), 277-283.

[18] Weerakoon, S. (1998). Complex inversion formula for Sumudu transforms. International Journal of Mathematical Education in Science and Technology, 29(4), 618-621.

[19] Eltayeb, H., K1licman, A., A note on double Sumudu transform and double Laplace transform. International Journal of Pure and Applied Mathematics, to appear.

[20] Eltayeb, H., Kilıcman, A., \& Fisher, B. (2010). A new integral transform and associated distributions. Integral Transforms and Special Functions, 21(5-6), 367-379.

[21] Belgacem, F.C.M., Karaballi, A.A., \& Kalla, S.L. (2003). Analytical investigations of the Sumudu transform and applications to integral production equations. Mathematical Problems in Engineering, 3, 103-118.

[22] Kılıçman, A., Eltayeb, H., \& Agarwal, R.P. (2010). On Sumudu transform and system of differential equations. Abstract and Applied Analysis, vol. 2010, Article ID 598702, 11 pages.

[23] Patel T., \& Meher, R. (2016). Adomian decomposition Sumudu transform method for convective fin with temperature- dependent internal heat generation and thermal conductivity of fractional order energy balance equation. International Journal of Applied and Computational Mathematics, 2(2), 1-17.

[24] Patel T., \& Meher, R. (2016). A study on fin efficiency analysis of convective straight fins with temperature dependent thermal conductivity by using adomian decomposition Sumudu transform method. International Journal of Pure and Applied Mathematics, 110(2), 311-326. 